\title{
Optimized Local Ternary Patterns: A New Texture Model with Set of Optimal Patterns for Texture Analysis
}

\author{
${ }^{1}$ Madasamy Raja, G. and ${ }^{2}$ V. Sadasivam \\ ${ }^{1}$ Department of Computer Science and Engineering, \\ MET Engineering College, Chenbagaramanputhoor, Tamilnadu-629304, India \\ ${ }^{2}$ Manonmaniam Sundaranar University, Tirunelveli, Tamilnadu-627012, India
}

Received 2012-08-30, Revised 2012-12-10; Accepted 2013-01-22

\begin{abstract}
Texture analysis is one of the important as well as useful tasks in image processing applications. Many texture models have been developed over the past few years and Local Binary Patterns (LBP) is one of the simple and efficient approach among them. A number of extensions to the LBP method have been also presented but the problem remains challenging in feature vector generation and comparison. As textures are oriented and scaled differently, a texture model should effectively handle grey-scale variation, rotation variation, illumination variation and noise. The length of the feature vector in a texture model also plays an important role in deciding the time complexity of the texture analysis. This study proposes a new texture model, called Optimized Local Ternary Patterns (OLTP) in the spatial methods of texture analysis. The proposed texture model is based on Local Ternary Patterns (LTP), which in turn is based on LBP. A new concept called "Level of Optimality" to select the optimal set of patterns is discussed in this study. This proposed texture model uses only optimal patterns to extract the textural information from the digital images and thereby reducing the length of the feature vector. This proposed model is robust to image rotation, grey-scale transformation, histogram equalization and noise. The results are compared with other widely used texture models by applying classification tests to variety of texture images from the standard Brodatz texture database. Experimental results prove that the proposed texture model is robust to grey-scale variation, image rotation, histogram equalization and noise. Experimental results also show that the proposed texture model improves the classification accuracy and the speed of the classification process. In all tested tasks, the proposed method outperforms the earlier methods.
\end{abstract}

Keywords: G-Statistic Similarity Measurement, Level of Optimality, Local Binary Patterns (LBP), Local Ternary Patterns (LTP), Texture Classification, Transition Length

\section{INTRODUCTION}

Image analysis is a process that extracts useful information from the digital images. Image segmentation, image classification, image correspondence, image compression are some examples for image analysis. Feature extraction is an important step in almost every image analysis tasks. Feature extraction is a sub-process in image analysis that extracts some important features like color, texture and shape from a digital image. Among many features, texture plays a vital role in many image analyses, due to following reasons:

- It contains more information compared to other features

- It is an inherent and easy to recognize property of all surfaces

- Majority of the natural surfaces exhibit texture

Textures are characterized by spatial distributions of image pixel values in a local neighborhood region. Corresponding Author: Madasamy Raja, G., Department of Computer Science and Engineering, MET Engineering College, Chenbagaramanputhoor, Tamilnadu-629304, India 
Though there is no unique definition of texture because of its wide variability, somehow it can be defined as a structure composed of a large number of more or less ordered similar elements or patterns. As textures are having different dimensions, single method of texture model is not adequate for a variety of textures. Image analysis that is based on texture feature is known as texture analysis. Texture analysis is a process of extracting information from the texture images, which characterizes the spatial variations within the image by using mathematical procedures and models. Texture analysis generally falls into four general categories and they are statistical methods, geometrical methods, model-based methods and signal processing methods (Tuceryan and Jain, 1999).

\subsection{Literature Review and Related Work}

In the digital images, the spatial distributions of grey values decide the textural features and hence, statistical methods analyze the spatial distribution of pixel values in the digital image. Based on the number of pixels defining the local feature, statistical methods can be classified into first-order statistical methods, second-order statistical methods and higher-order statistical methods (Ojala and Pietikyinen, 2004). A large number of statistical texture approaches have been proposed, ranging from first order statistics to higher order statistics. As first order statistical methods cannot model the texture perfectly, higher order statistics are widely used for texture analysis (Moasheri and Azadinia, 2011). Grey level cooccurrence matrices (Haralick et al., 1973), grey level differences (Weszka et al., 1976) and Local Binary Patterns (Ojala et al., 1996) are some of the popular second-order statistical texture methods for texture analysis. Galloway (1975) and after some years Tsatsanis and Giannakis (1992) have proved that at the cost of computational complexity, higher than second-order statistical methods could also be used for statistical texture analysis.

Geometrical methods are based on the concept that texture could be considered as a spatial organization of texture primitives. Fu (1982) proposed an idea in which the texture image is viewed as texture primitives, which are arranged according to a placement rule and texture analysis is a process of identifying those primitives or the placement rule. Matsuyama et al. (1983) used Fourier spectrum of a texture image to detect texture periodicity for the texture analysis. Liu et al. (2005) examined the structures of texture patterns in terms of their translation symmetries for the texture analysis.

In model-based methods, mathematical models are used to represent the textures in an image such as fractals (Xia et al., 2006), random field models (Zhu et al., 1998) and so on. Signal processing methods consider the frequency domain of the digital images for the texture feature extraction. Coggins and Jain (1985) tested multichannel filtering approach using frequency and orientation selective filters for the texture analysis. Under signal processing methods, usage of Gabor filters (Daugman, 1980) and pyramids (Heeger and Bergen, 1995) have also been successfully investigated.

\subsection{Local Binary Patterns (LBP)}

The texture model Local Binary Patterns was first developed by Ojala et al. (1996). For a $3 \times 3$ neighborhood around a centre pixel in an image, the LBP operator is defined as Equation 1:

$$
\begin{aligned}
& \operatorname{LBP}_{8}=\sum_{n=1}^{8} 2^{n} s\left(i_{n}-i_{c}\right) \\
& \text { and } s(u)=\left\{\begin{array}{l}
1 \text { if } u \geq 0 \\
0 \text { otherwise }
\end{array}\right.
\end{aligned}
$$

where, $\mathrm{n}$ is the number of pixels in the considered neighborhood, $i_{c}$ is the pixel value of center pixel $c$ and $i_{n}$ are the pixel values of $n$. This LBP operator considers a local neighborhood with a certain radius around every pixel in the image and all the neighboring pixels are encoded by thresholding against the centre pixel of the neighborhood by using the piecewise function $\mathrm{s}(\mathrm{u})$. Then all the encoded neighboring labels are concatenated to form a binary pattern string and finally the histogram of all these binary pattern strings is used as the texture descriptor.

Ojala et al. (2002) extended their earlier work in the name of Uniform Local Binary Patterns (ULBP) by introducing a new concept called "Uniform patterns". By considering the uniform patterns, total number of patterns in LBP is reduced from 256 to 58 and it is observed that in a texture image, for a $3 \times 3$ neighborhood, nearly $90 \%$ of encoded labels are uniform patterns only.

Even after ten years of its introduction, still there have been various extensions and modifications from the original LBP operator, because it is computationally simple and very robust in terms of rotational and gray-scale variations. Some recent developments in medical imaging (Liu et al., 2011), moving object detection (Trefny and Matas, 2010) and facial expression recognition (Ahmed et al., 2011) prove that the LBP texture model is still receiving a lot of attention. However LBP texture model is considered to be sensitive to noise especially in uniform regions (Rodriguez and Marcel, 2006). Moreover, it supports only a binary level comparison for encoding and thereby it is inadequate to represent the local texture information in more detail.

\subsection{Local Ternary Patterns (LTP)}

As LBP may be sensitive to noise, a 3-valued pattern instead of a binary pattern was introduced by Tan and 
Triggs (2010). When a $3 \times 3$ neighborhood around a centre pixel in an image is considered, the LTP operator takes the form Equation 2:

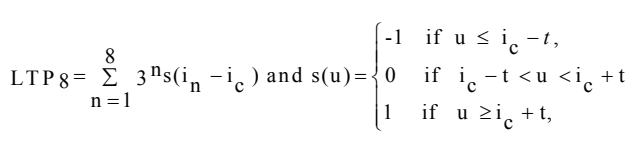

where, $\mathrm{t}$ is a user-defined threshold, $\mathrm{n}$ is the number of neighboring pixels surrounding the center pixel $c, i_{c}$ is the pixel value of $c$ and $i_{n}$ are the pixel values of $n$. In LTP, pattern strings are formed with three values $(-1,0,1)$ according to a predefined and fixed threshold $t$, the length of the pattern histogram is very high $\left(3^{8}\right)$. To reduce the dimension of the pattern histogram, the ternary pattern is converted into binary pattern, by splitting it into its positive and negative parts. Two separate histograms for both positive and negative components are calculated and then the results are concatenated.

A three valued LBP was also proposed to resist the noise with the help of fuzzy logic and a soft histogram (Ahonen and Pietikainen, 2007). Another three valued LBP version in the name of Local Texture Patterns was also introduced (Suruliandi and Ramar, 2008), in which all the three-valued patterns are considered as it is and there is no splitting of the patterns for the purpose of dimensionality reduction of the pattern spectrum. In Local Texture Patterns method, patterns with a maximum of three spatial transitions are considered as uniform patterns and the dimension of the pattern spectrum was 46. The same Local Texture Patterns method was modified in the name of Ternary Pattern Operator by reducing the uniformity measure to two transitions, with the pattern spectrum dimension of 24 (Suguna and Anandhakumar, 2010). Both Local Texture Patterns and Ternary Pattern Operator methods were successfully tested for texture classification.

Nanni et al. (2010) classified the pain states from facial expressions, by using extensions of LTP, namely Elongated Ternary Patterns (ELTP) and Improved Local Ternary Patterns (ILTP). In another extension, the combination of LTP with Histograms of Gradients (HOG) was successfully tested for object detection (Hussain and Triggs, 2010). The noise resistivity of Local Ternary Patterns has been again successfully proved in the form of Extended Local Ternary Patterns by Liao (2010). Center-symmetric Local Ternary Patterns, another novel texture feature based on LTP, was also presented for pedestrian detection (Zheng et al., 2011). The major advantage of LTP is it is resistant to noise but at the same time the major disadvantage of LTP and its modifications is that they are not invariant under grey-scale transform of intensity values as its encoding is based on a fixed predefined thresholding. Hence the objective of this research is to propose a new texture model as an extension of LTP, which takes the advantages of both LBP and LTP, to accomplish a better tolerance against grey-scale variation, histogram equalization, image rotation and noise, while at the same time optimizing the length of the patterns histogram.

\section{MATERIALS AND METHODS}

\subsection{Encoding Method of the Proposed Texture Model}

In this study, a theoretically and computationally simple but efficient new texture model called Optimized Local Ternary Patterns (OLTP) is proposed. The proposed texture model OLTP uses only limited number of uniform patterns namely optimal patterns and thereby maintains the length of the pattern histogram as optimum.

This new texture model considers a $3 \times 3$ local neighborhood region in a digital image for texture feature extraction. Let $i_{c}, i_{1}, i_{2}, \ldots, i_{8}$ be the pixel values of a local $3 \times 3$ neighborhood region where $i_{c}$ is the value of the central pixel and $i_{1}, i_{2}, \ldots, i_{8}$ are the pixel values of its 8 neighbors. In this proposed texture model, the pattern string value for the local $3 \times 3$ neighborhood region is defined as Equation 3:

$$
P\left(i_{c}, i_{n}\right)=\left\{\begin{array}{cc}
0 & \text { if } i_{n}<(1-\sigma) i_{c} \\
1 & \text { if }(1-\sigma) i_{c} \leq i_{n} \leq(1+\sigma) i_{c} \\
5 & \text { if } i_{n}>(1+\sigma) i_{c}
\end{array}\right.
$$

where, $\sigma$ is a small scaling factor and it is assigned as 0.05 . Figure 1 shows how the pattern value for a $3 \times 3$ local neighborhood is calculated using Equation 3 . Figure 1a represents a sample $3 \times 3$ local neighborhood which is to be encoded into pattern matrix by using Equation 3 and Fig. 1b contains the corresponding encoded pattern matrix. The pattern string for the selected $3 \times 3$ neighborhood region is given in Fig. 1c which is obtained from Fig. 1b by concatenating the encoded values in the pattern matrix.

For a $3 \times 3$ local neighborhood region, the total number of different pattern strings will be $6561\left(3^{8}\right)$. In other words, Equation. 3 will deliver any one pattern string from a set of 6561 different pattern strings for a $3 \times 3$ local neighborhood region. So, a complete texture image can be described by a pattern histogram of 6561 bins that represents the occurrence frequency of pattern strings over the texture image. Among these 6561 different pattern strings, it is observed that only few pattern strings are frequently occurring patterns and all other pattern strings are very rarely occurring in a small local neighborhood of a texture image. So keeping separate bins for the rarely occurring pattern strings is a futile exercise which may result in wastage of memory as well as wastage of time. 

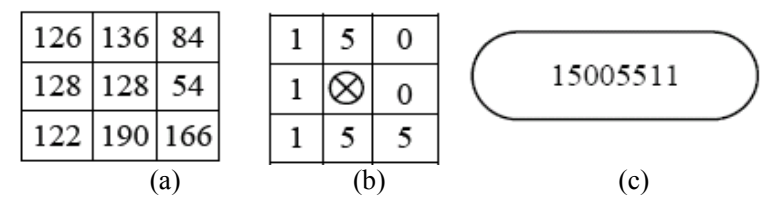

(c)

Fig. 1. Method for encoding the Patterns in OLTP texture model (a) $3 \times 3$ local region, (b) pattern matrix, (c) pattern string

Middle level region (1)

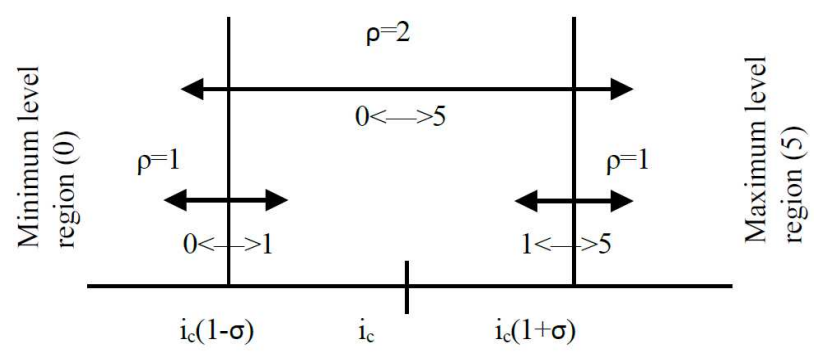

Fig. 2. A logical view of calculating Transition Length ( $\rho$ ) among the sub patterns in the uniform pattern strings

Further, the analysis of these frequently occurring pattern strings found that all these pattern strings have uniform circular structure and they are rotation invariant. To identify these frequently occurring pattern strings which are rotation invariant, let us consider, a uniformity measure ' $U$ ' which corresponds to the number of spatial transitions circularly among the sub patterns in the pattern strings. The uniformity measure ' $U$ ' is defined as Equation 4:

$\mathrm{U}=\mathrm{F}\left(\mathrm{P}\left(\mathrm{i}_{\mathrm{c}}, \mathrm{i}_{1}\right), \mathrm{P}\left(\mathrm{i}_{\mathrm{c}}, \mathrm{i}_{8}\right)\right)+\sum_{n=2}^{8} \mathrm{~F}\left(\mathrm{P}\left(\mathrm{i}_{\mathrm{c}}, \mathrm{i}_{\mathrm{n}}\right), \mathrm{P}\left(\mathrm{i}_{\mathrm{c}}, \mathrm{i}_{\mathrm{n}-1}\right)\right)$
where $\quad \mathrm{F}(\mathrm{A}, \mathrm{B})= \begin{cases}1 & \text { if }|\mathrm{A}-\mathrm{B}|>0 \\ 0 & \text { otherwise }\end{cases}$

For example, the pattern string 55555555 has U value of 0 , the pattern string 55555511 has $U$ value of 2 and the pattern string 11105551 has $U$ value of 3 . As it is already observed that frequently occurring pattern strings are having only few spatial transitions among the sub patterns, this research puts a constraint on the pattern strings to contain a maximum of 3 spatial transitions $(\mathrm{U} \leq 3)$. Those frequently occurring pattern strings which satisfy the condition of $\mathrm{U} \leq 3$ are termed as uniform pattern strings and they are rotational invariant too. For $\mathrm{U}=0$, there are 3 uniform pattern strings and there is no uniform pattern string exist with $U=1$. For $U=2$, there are 21 uniform pattern strings and for $U=3$, another 21 uniform pattern strings are available. In total there are 45 uniform pattern strings available, when $U \leq 3$ is considered as a constraint for the uniformity measure. All these 45 uniform pattern strings are having a maximum of 3 transitions (" $0<->1$ " or " $1<->5$ " or " $0<->5$ ") between the successive encoded values (sub patterns).

\subsection{Transition Length ( $\rho)$ of the sub patterns}

Before going into the details of Level of Optimality $\left(\mathrm{L}_{\text {opt }}\right)$, it is necessary to introduce a concept called transition length $(\rho)$ of successive encoded values (sub patterns) in the uniform pattern strings. Figure 2 illustrates the basic idea behind the calculation of transition length $(\rho)$ among the sub patterns in the uniform pattern strings. In Fig. 2, $i_{c}$ represents the value of the centre pixel in a $3 \times 3$ local neighborhood region, for which the uniform pattern string is encoded. Minimum level region represents those sampling points around the centre pixel $\left(i_{c}\right)$ which are encoded as 0 , because they fall below to the minimum threshold $\left(i_{c} *(1-\sigma)\right)$. Maximum level region represents those sampling points around the centre pixel $\left(i_{c}\right)$ which are encoded as 5 , because they fall above to the maximum threshold $\left(\mathrm{i}_{\mathrm{c}} *(1+\sigma)\right)$. Middle region represents the surrounding pixels of a local $3 \times 3$ neighborhood, which are encoded as 1 , because they fall between the minimum and maximum threshold regions.

In the uniform pattern strings, when a spatial transition occurs between the neighboring regions, then the length of that transition is considered as 1 . For example, the sub pattern ' 01 'in the uniform pattern string represents a spatial transition between minimum level region and middle level region. The sub pattern ' 15 ' of uniform pattern strings represents a spatial transition between the middle level region and maximum level region. In both these cases, the transition length is considered as 1 since the spatial transitions occur between the neighboring regions. When there is a spatial transition between the extreme regions, then the transition length is considered as 2 . For example, the sub pattern ' 05 ' in the uniform pattern string represents a spatial transition between minimum level region and maximum level region for which the transition length is assigned as 2. Further, those patterns which are having two consecutive transitions among the sub patterns, transition lengths are assigned according to the flow direction of the spatial transitions. Table 1 contains the transition lengths of various sub patterns that are available in the uniform pattern strings.

\subsection{Level of Optimality $\left(\mathrm{L}_{\mathrm{opt}}\right)$}

This research introduces a new concept called "Level of Optimality" to check, whether a particular uniform pattern string can be included into the optimal uniform pattern set or not. Thus the concept "Level of Optimality" is acting as a tool to filter out the suboptimal uniform pattern strings which are seldom active in the process of texture feature extraction. 


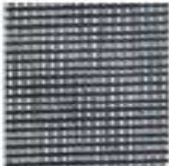

(a)

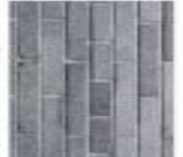

(b)

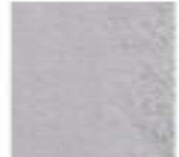

(c)

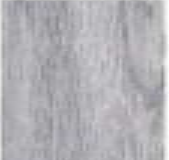

(d) (a)

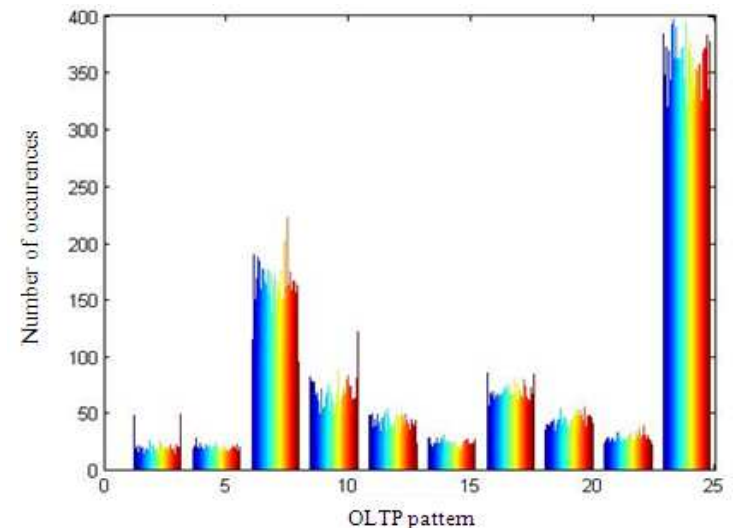

(e)

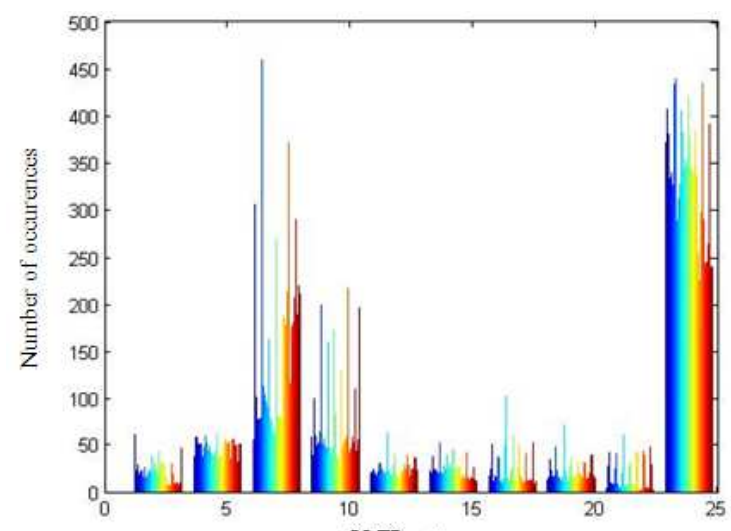

(f)

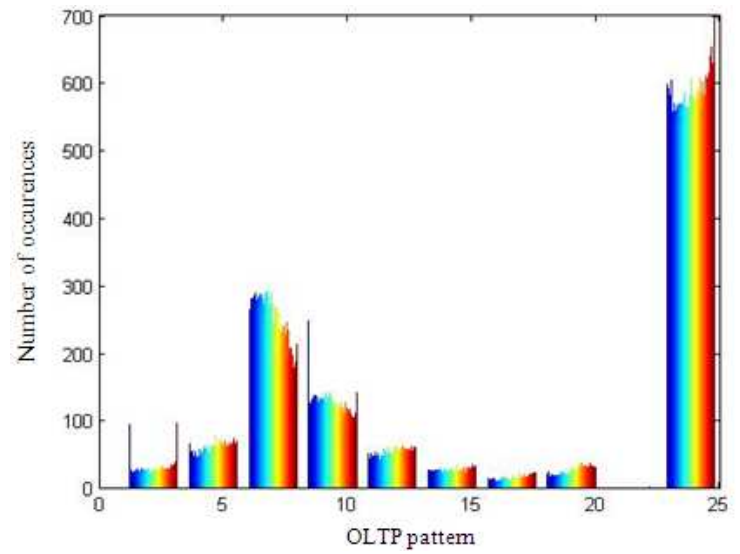

(g)

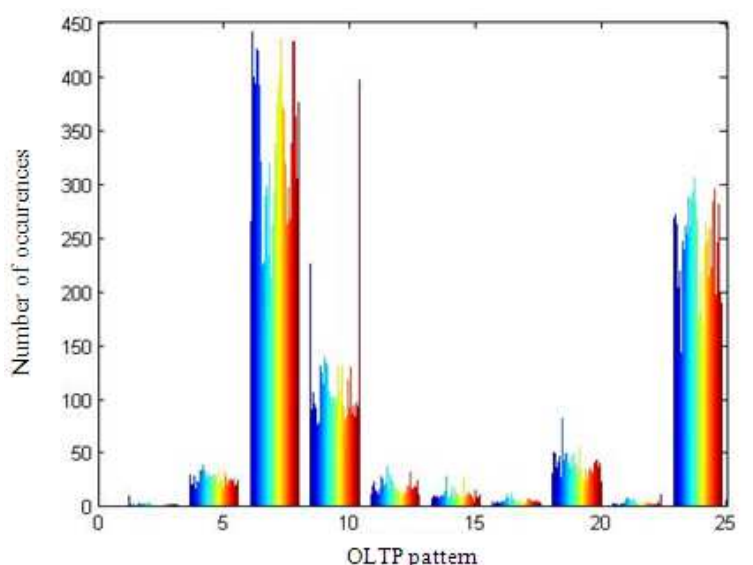

(h)

Fig. 3. Selected Images and their corresponding pattern spectrum obtained through OLTP texture model (a) Images: AFrench canvas, B-Brick, C-Water, D-Wood grain

Table 1. Shows the details of the Transition Length $(\rho)$ for the sub patterns of uniform pattern strings

Transition length

\begin{tabular}{|c|c|}
\hline Sub pattern type & Transition length $(\rho)$ \\
\hline '01' & 1 \\
\hline ‘ $10 ’$ & 1 \\
\hline ' 15 ' & 1 \\
\hline ' 51 ' & 1 \\
\hline '05' & 2 \\
\hline$' 50 ’$ & 2 \\
\hline$' 015$ ' & 1 \\
\hline '510' & 1 \\
\hline ' 105 ' & 2 \\
\hline ' 150 ' & 2 \\
\hline '051' & 2 \\
\hline '501' & 2 \\
\hline
\end{tabular}

Table 2. Shows some pattern strings used in the proposed texture model with their details

Details of pattern strings

\begin{tabular}{lllll} 
Pattern string & 'U'value & $\begin{array}{l}\text { Level of } \\
\text { optimality }\end{array}$ & $\begin{array}{l}\text { Uniform } \\
\text { pattern }\end{array}$ & $\begin{array}{l}\text { Optimal } \\
\text { pattern }\end{array}$ \\
\hline 00000000 & 0 & 2 & Yes & Yes \\
00000555 & 2 & 2 & Yes & Yes \\
00000005 & 2 & 1 & Yes & No \\
01010101 & 8 & 5 & No & No \\
00000150 & 3 & 1 & Yes & No \\
00001550 & 3 & 2 & Yes & Yes \\
\hline
\end{tabular}

Obviously this concept "Level of Optimality" is also useful in reducing the dimension of the pattern histogram which is a deciding factor in the execution time of almost all texture analysis.

The elements of the uniform pattern strings are either 0 or 1 or 5 or any combinations of these values. If the number of occurrences (cardinality) of 0,1 and 5 are 
represented by card (0), card (1) and card (5) respectively, then the level of optimality for a particular uniform pattern string can be computed as Equation 5:

$\mathrm{L}_{\text {opt }}=\frac{1+\min \{\operatorname{card}(0), \operatorname{card}(1), \operatorname{card}(5)\}}{\max (\rho)}$

where, $\operatorname{card}(0), \operatorname{card}(1)$ and $\operatorname{card}(5) \neq 0$ and $\rho$ refers to the transition length among the sub patterns in the uniform pattern strings. For the patterns strings, which are not having any spatial transitions among the sub patterns, transition length cannot be calculated and obviously the level of optimality for these kinds of patterns also cannot be decided. Hence, those patterns strings with no spatial transitions are assigned with optimality level 2 by default so that they are considered as optimal patterns.

\subsection{Description of an Optimal Pattern}

A uniform pattern is said to be an optimal pattern, if it satisfies the following criteria:

- The pattern string must not contain more than 3 transitions between the successive encoded values (sub patterns) in the pattern string

- The level of optimality must be greater than or equal to 2

\subsection{Optimized Local Ternary Patterns (OLTP)}

The following texture model, Optimized Local Ternary Patterns (OLTP) which is rotational invariant, gray-scale invariant, image histogram equalization invariant and noise resistant is proposed. OLTP operator uses only optimal set of patterns for describing a local image texture Equation 6:

OLTP $=\left\{\begin{array}{c}\sum_{\mathrm{i}=1}^{8} \mathrm{p}\left(\mathrm{i}_{\mathrm{c}}, \mathrm{i}_{\mathrm{n}}\right) \text { if } \mathrm{U} \leq 3 \text { and } \mathrm{L}_{\text {opt }} \geq 2 \\ 25 \text { otherwise }\end{array}\right.$

This newly proposed texture model, OLTP uses a total number of 24 unique optimal patterns for texture representation. All other patterns are termed as "suboptimal" patterns and grouped under one label 25. Therefore the dimension of pattern spectrum has been reduced from 6561 to 25 , that too with optimal set of patterns. Among these 24 unique optimal patterns, 17 patterns are having a maximum of 2 transitions in their sub patterns $(U=2), 3$ patterns are having $U=0$ and there are 4 patterns with $U=3$. Table 2 shows some of the pattern strings with relevant details of their uniformity, level of optimality $\left(\mathrm{L}_{\mathrm{opt}}\right)$ and whether they are optimal patterns or not. Figure 3a shows some selected texture images from Brodatz album (Brodatz, 1966) and Figure 3b shows their corresponding pattern spectrum of the optimal patterns obtained through proposed OLTP texture model.

\subsection{Experimental Setup}

In this study, pattern spectrum of the optimal patterns is used as a tool for checking the similarity between sample and model textures. The non-parametric similarity measure G-statistic (Sokal and Rohlf, 2009) is used in the classification algorithm to test the similarity between two different pattern spectrums. This similarity measure G-statistic is also known as log-likelihood ratio which is computed as Equation 7:

$$
\begin{aligned}
& G=2\left[\sum_{s, m} \sum_{i=1}^{n} f_{i} \log f_{i}\right]-\left[\sum_{s, m}\left(\sum_{i=1}^{n} f_{i}\right) \log \left(\sum_{i=1}^{n} f_{i}\right)\right] \\
& -\left[\sum_{i=1}^{n}\left(\sum_{s, m} f_{i}\right) \log \left(\sum_{s, m} f_{i}\right)\right] \\
& \left.+\left[\sum_{s, m} \sum_{i=1}^{n} f_{i}\right] \log \left(\sum_{s, m} \sum_{i=1}^{n} f_{i}\right)\right]
\end{aligned}
$$

where, $\mathrm{s}$ is the pattern histogram of the optimal patterns of the test sample and $\mathrm{m}$ is the pattern histogram of the optimal patterns of the model sample, $n$ is the total number of optimal patterns in the OLTP texture model and $\mathrm{f}_{\mathrm{i}}$ is the frequency of a particular optimal pattern i. In classification, a particular test sample is classified to the model sample to which it is having a high probability of belonging to the same population. This probability is measured by the value of similarity measure G-statistic. Lower the values of the similarity measure, higher the possibilities that both model and test sample images are likely to have come from the same population. If the value of G-statistic is high, it means that pattern histograms of model sample and test sample are variable and they are not same. This proposed method uses the K-nearest neighbor classification algorithm for texture classification experiments.

\section{RESULTS}

\subsection{Statistics of Distribution of Optimal Patterns in OLTP}

For the study of the statistics of distribution of optimal patterns in the texture images, some widely used texture images are chosen from the standard Brodatz texture database. For all these selected texture images, the distribution of the 24 numbers of optimal patterns of the proposed OLTP texture model and the distribution of 45 numbers of uniform patterns of LTP texture model are calculated and listed in Table 3. By comparing the values in Table $\mathbf{3}$, it is observed that there is only marginal difference exists between the distribution of the optimal patterns of OLTP and uniform patterns of LTP even when the number of 
patterns has been reduced to almost half in quantity in the proposed texture model OLTP compared to LTP. In the practical applications, this marginal difference in the distribution of patterns becomes negligible which is going to be proved in the following experiments.

\subsection{Texture Classification Using Proposed Model OLTP}

This research uses texture classification process to check the validity of the proposed texture model (OLTP). For all the experiments, both model samples as well as test samples are extracted from the selected texture images in various sizes.

\subsection{Experiment \#1:}

The Brodatz texture images shown in Fig. 3a of size $640 \times 640$ were taken for this experiment. Each individual texture image is considered as a model sample and there are 4 model samples in total. The selected texture images are resized into $256 \times 256$ and 65536 test samples were extracted in three different sizes. Each test sample is compared against the model samples using Equation 7 and the test sample is classified to the nearest neighbor model sample for which it has a minimum distance (G-Statistics value). The classification is performed for test samples of size of $10 \times 10,20 \times 20$ and $30 \times 30$. Table 4 shows the classification performance of the proposed texture model and from the table, it is observed that the classification results are excellent with high classification accuracy. Another observation from the classification results is that the classification accuracy increases as the sample size increases.
With the size of $15 \times 15,500$ test samples were again extracted from each texture class of the images of Fig. 3a for the comparative analysis of the classification accuracy of the proposed texture model OLTP. In this sub experiment, for four different texture classes there were 2000 test samples in total. Already existing texture models Texture Spectrum (TS) and LBP are considered along with the proposed texture model for this comparative study. The result of the comparison is given in the form of a chart in Fig. 4. In the case of TS texture model, 89.29 percent average classification accuracy is achieved. Though TS model is considered as a good texture model, it uses very large number of bins in the pattern histogram (6561) and took more execution time. The LBP model gave an average classification accuracy of 83.9 percent. Even though LBP texture model gave moderate results, the advantage of this model is, it uses only 10 bins in the pattern histogram with lower computational complexity. Among the three texture models under consideration, the proposed OLTP texture model delivers superior performance with the average classification accuracy of 98.7 percent.

\subsection{Experiment \#2:}

Experiment \#2 has been carried out to study the rotation invariance property of the proposed OLTP approach. Four different texture image classes at 6 different rotation angles $\left(0^{\circ}, 30^{\circ}, 60^{\circ}, 90^{\circ}, 120^{\circ}\right.$ and $\left.150^{\circ}\right)$ are chosen from the Brodatz album for this study. Hence, there are 6 images in each texture class and totally 24 different texture images are considered, which are displayed in Fig.

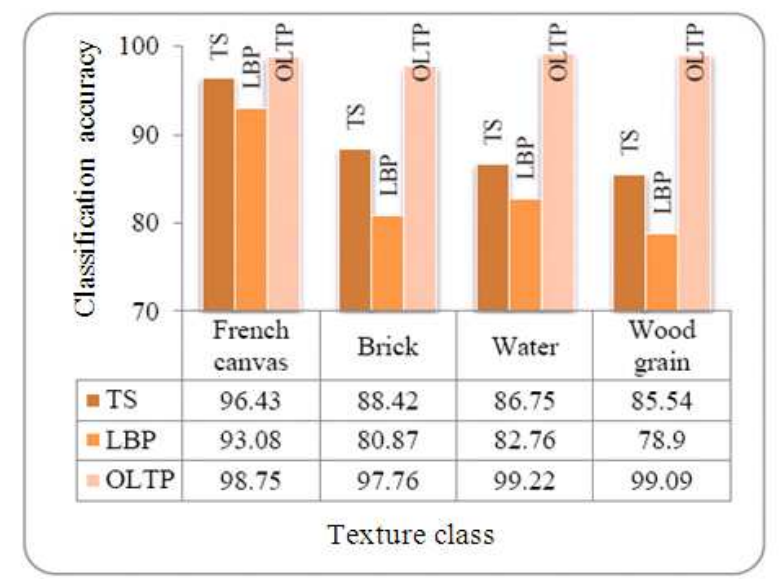

Fig. 4. Comparative analysis of the classification accuracy of various texture models 


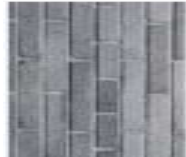

(a1)

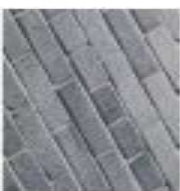

(a2)

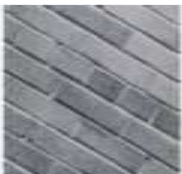

(a3)

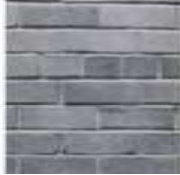

(a4)

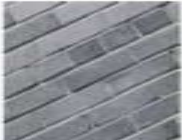

(a5)

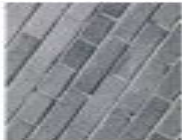

(a6)

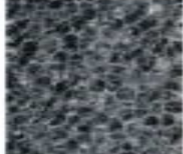

(b1)

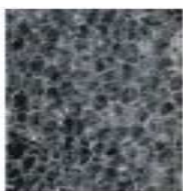

(b2)

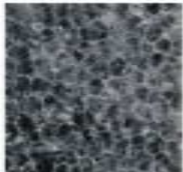

(b3)

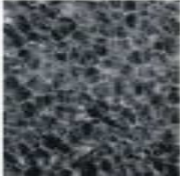

(b4)

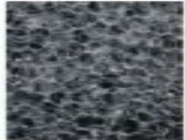

(b5)

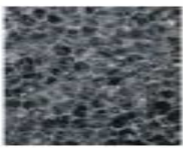

(b6)

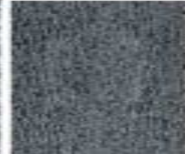

(c1)

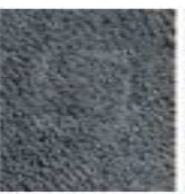

(c2)

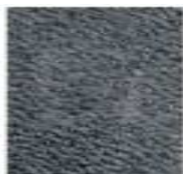

(c3)

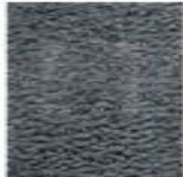

(c4)

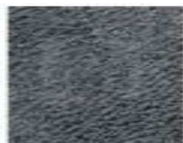

(c5)

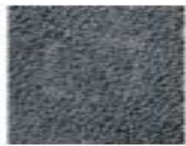

(c6)

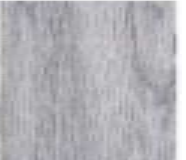

(d1)

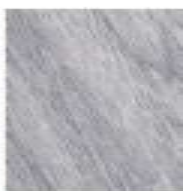

(d2)

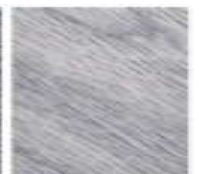

(d3)

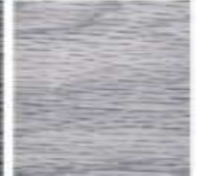

(d4)

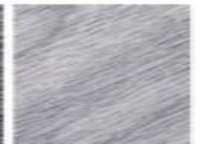

(d5)

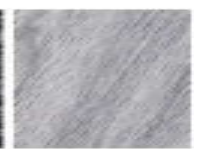

(d6)

Fig. 5. Sample images used in Experiment \#2 with their rotation angles mentioned with in parenthesis (a1) Brick(00), (a2) $\operatorname{Brick}\left(30^{\circ}\right)$, (a3) Brick $(600)$, (a4) Brick $\left(90^{\circ}\right)$, (a5) Brick $\left(120^{\circ}\right)$, (a6) Brick $\left(150^{\circ}\right)$, (b1) Bubbles $\left(0^{\circ}\right)$, (b2) Bubbles $\left(30^{\circ}\right)$, (b3) Bubbles $\left(60^{\circ}\right)$, (b4) Bubbles $\left(90^{\circ}\right)$, (b5) Bubbles $\left(120^{\circ}\right)$, (b6) Bubbles $\left(150^{\circ}\right)$, (c1) Leather $\left(0^{0}\right)$, (c2) Leather $\left(30^{\circ}\right)$, (c3) Leather(600), (c4) Leather $\left(90^{\circ}\right),(\mathrm{c} 5)$ Leather $\left(120^{\circ}\right),(\mathrm{c} 6)$ Leather(1500), (d1) Wood(00), (d2) Wood(300), (d3) Wood(600), (d4) Wood $(900),(d 5) \operatorname{Wood}\left(120^{\circ}\right),(d 6) \operatorname{Wood}\left(150^{\circ}\right)$

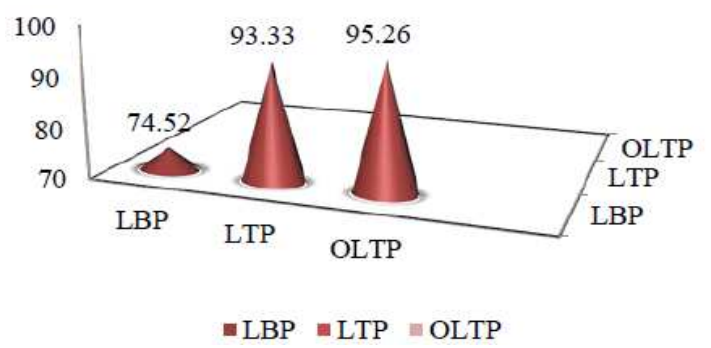

Fig. 6. Performance evaluation on the basis of average classification accuracy (\%) of various texture models for the selected rotated texture images 


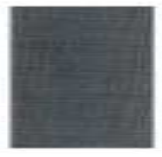

(a)

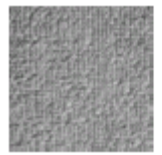

(f)

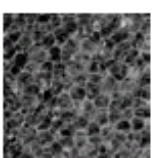

(k)

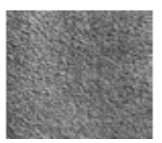

(p)

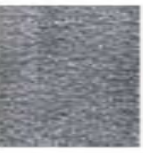

(b)

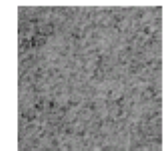

$(\mathrm{g})$

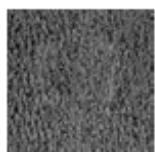

(1)

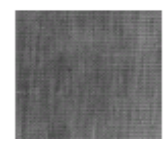

(q)

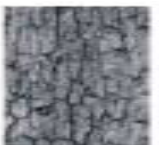

(c)
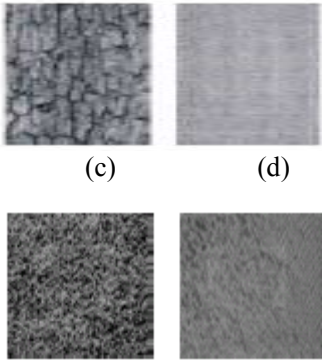

(h)

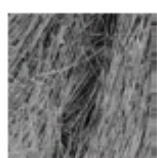

(m)
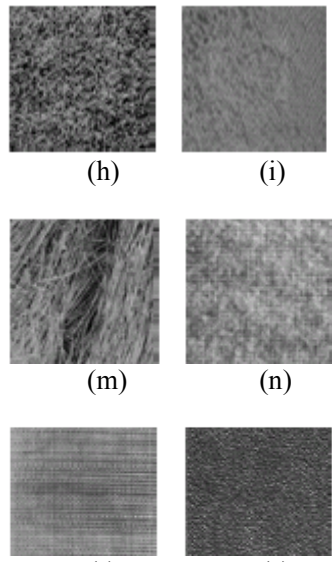

(r) (d)

(i)

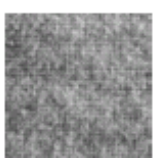

(n)

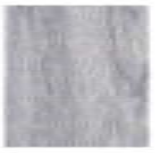

(e)

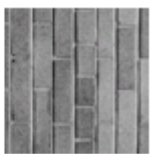

(j)

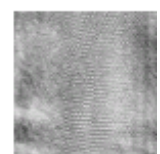

(o)

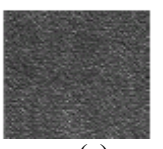

(s)

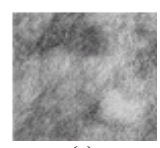

(t)

Fig. 7. Sample images used in Experiment \#3 (a) French Canvas, (b) Pigskin, (c) Bark, (d) Weave, (e) Wood, (f) Raffia, (g) Sand, (h) Grass, (i) Water, (j) Brick, (k) Bubbles, (l) leather, (m) Straw, (n) Wool, (o)Reptile skin, (p) Pressed cork, (q) Cloth, (r) Matting, (s) Paper, (t) Fur

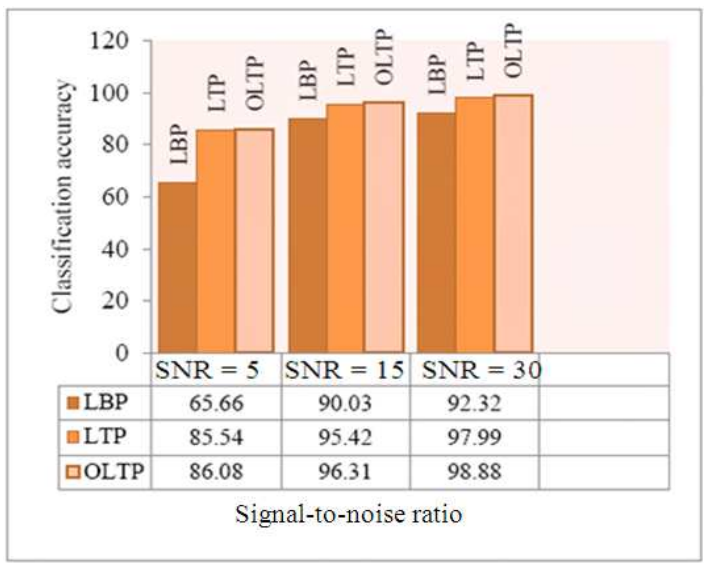

Fig. 8. Average classification accuracy (\%) for the texture images which were corrupted by additive Gaussian noise with various SNR values

Table 3. Shows the distribution of patterns in OLTP and LTP texture models for various texture classes

\begin{tabular}{llr}
\hline $\begin{array}{l}\text { Proportion } \\
\text { of patterns } \\
\text { Texture class }\end{array}$ & Distribution of patterns (\%) \\
\hline French canvas & LTP & OLTP \\
Brick & 62 & 60 \\
Water & 52 & 51 \\
Bark & 73 & 41 \\
Straw & 42 & 41 \\
Weave & 44 & 40 \\
Wood & 42 & 71 \\
Wool & 71 & 41 \\
Pigskin & 43 & 42 \\
\hline
\end{tabular}


Table 4. Classification of the test images using OLTP texture model

Details of the classified samples

\begin{tabular}{|c|c|c|c|c|c|c|}
\hline & \multicolumn{6}{|c|}{ Classification results (Out of 65536 Samples) } \\
\hline & Sample size & French canvas & Brick & Water & Wood & $\begin{array}{l}\text { Classification } \\
\text { accuracy (\%) }\end{array}$ \\
\hline French & 10 & 63622 & 1568 & 303 & 43 & 97.08 \\
\hline \multirow[t]{2}{*}{ canvas } & 20 & 65424 & 85 & 15 & 12 & 99.83 \\
\hline & 30 & 65536 & 0 & 0 & 0 & 100.00 \\
\hline \multirow[t]{3}{*}{ Brick } & 10 & 1099 & 64117 & 234 & 86 & 97.83 \\
\hline & 20 & 132 & 65369 & 32 & 3 & 99.75 \\
\hline & 30 & 0 & 65536 & 0 & 0 & 100.00 \\
\hline \multirow[t]{3}{*}{ Water } & 10 & 35 & 11 & 64102 & 1388 & 97.81 \\
\hline & 20 & 15 & 0 & 65389 & 132 & 99.78 \\
\hline & 30 & 0 & 0 & 65536 & 0 & 100.00 \\
\hline \multirow[t]{3}{*}{ Wood } & 10 & 2 & 112 & 1345 & 64077 & 97.77 \\
\hline & 20 & 0 & 10 & 168 & 65358 & 99.73 \\
\hline & 30 & 0 & 0 & 0 & 65536 & 100.00 \\
\hline \multirow[t]{3}{*}{ Average } & 10 & & & & & 97.62 \\
\hline & 20 & & & & & 99.77 \\
\hline & 30 & & & & & 100.00 \\
\hline
\end{tabular}

Table 5. Classification of the rotated test images using various texture models

Details of the classification accuracy

\begin{tabular}{|c|c|c|c|c|c|c|c|}
\hline \multirow{2}{*}{$\begin{array}{l}\text { Texture } \\
\text { Model }\end{array}$} & \multicolumn{7}{|c|}{ Classification Accuracy for different degrees of rotation $(\%)$} \\
\hline & Texture class & $30^{\circ}$ & $60^{\circ}$ & $90^{\circ}$ & $120^{\circ}$ & $150^{\circ}$ & Average \\
\hline \multirow[t]{4}{*}{ LBP } & Brick & 74.32 & 63.14 & 52.53 & 54.110 & 59.570 & 60.73 \\
\hline & Bubbles & 69.21 & 51.47 & 60.44 & 59.180 & 58.120 & 59.68 \\
\hline & Leather & 91.01 & 90.08 & 89.37 & 91.300 & 88.240 & 90.00 \\
\hline & Wood & 85.54 & 87.74 & 88.31 & 89.980 & 86.660 & 87.65 \\
\hline \multirow[t]{4}{*}{ LTP } & Brick & 82.43 & 91.44 & 90.66 & 93.770 & 92.400 & 90.14 \\
\hline & Bubbles & 82.45 & 90.12 & 92.46 & 90.090 & 95.380 & 90.10 \\
\hline & Leather & 100.00 & 100.00 & 100.00 & 100.00 & 100.00 & 100.00 \\
\hline & Wood & 92.77 & 91.15 & 95.22 & 90.390 & 95.850 & 93.08 \\
\hline \multirow[t]{4}{*}{ OLTP } & Brick & 84.05 & 93.42 & 92.68 & 94.620 & 94.110 & 91.78 \\
\hline & Bubbles & 86.63 & 91.04 & 95.64 & 92.630 & 98.230 & 92.83 \\
\hline & Leather & 100.00 & 100.00 & 100.00 & 100.00 & 100.00 & 100.00 \\
\hline & Wood & 95.82 & 96.57 & 98.23 & 95.030 & 96.540 & 96.44 \\
\hline
\end{tabular}

Table 6. Classification performance analysis for the histogram-equalized Average classification accuracy for the various texture models

\begin{tabular}{|c|c|c|c|}
\hline \multirow[b]{2}{*}{$\begin{array}{l}\text { Texture } \\
\text { models }\end{array}$} & \multicolumn{3}{|c|}{ Classification accuracy (\%) } \\
\hline & $\begin{array}{l}\text { Original texture } \\
\text { images }\end{array}$ & $\begin{array}{l}\text { Histogram } \\
\text { Equalized } \\
\text { texture images }\end{array}$ & $\begin{array}{l}\text { Histogram Equalized } \\
\text { and randomly rotated } \\
\text { texture images }\end{array}$ \\
\hline LBP & 94.87 & 89.08 & 89.76 \\
\hline LTP & 96.85 & 91.64 & 90.80 \\
\hline OLTP & 97.77 & 95.42 & 92.79 \\
\hline
\end{tabular}

Table 7. Classification performance under the additive Gaussian noise environment Details of the classification accuracy for the various texture models

Classification accuracy (\%)

\begin{tabular}{|c|c|c|c|c|}
\hline Texture Models & $\mathrm{SNR}=100$ & $\mathrm{SNR}=35$ & $\mathrm{SNR}=15$ & $\mathrm{SNR}=5$ \\
\hline LBP & 92.32 & 91.21 & 88.43 & 61.33 \\
\hline LTP & 97.74 & 97.30 & 94.33 & 81.98 \\
\hline OLTP & 98.98 & 98.96 & 95.41 & 83.45 \\
\hline
\end{tabular}


In each texture class, 4 images are without any rotation(Fig. 5a1, b1, c1 and d1), 4 images are rotated at $30^{\circ}$ (Fig. 5a2,b2, c2 and d2), 4 images are rotated at $60^{\circ}$ (Fig. 5a3, b3, c3 and d3), 4 images are rotated at $90^{\circ}$ (Fig. 5a4, b4, c4 and d4), 4 images are rotated at $120^{\circ}($ Fig.5 a5, b5, c5 and d5) and 4 images are rotated at $150^{\circ}$ (Fig. 5a6, b6, c6 and d6). This experimental setup is somewhat tough because model samples are given without any rotation that is with $0^{\circ}$ rotations and the classification is tested with test samples with all other 5 rotation angles. With the sample size of $25 \times 25,100$ samples were extracted from every texture image and there were 2400 test samples in total which are taken for this experiment. Table 5 presents the classification performance of various texture models namely LBP, LTP and OLTP for the test samples. Figure 6 shows the comparative analysis of the average classification accuracy of the selected texture models for this experiment. From the results, it can be understood that even under the difficult experimental setup, the proposed texture method OLTP outperforms all other compared texture models by achieving the highest average classification accuracy of $95.26 \%$.

\subsection{Experiment \#3}

Experiment \#3 has been performed to study the image histogram equalization invariance property of the proposed OLTP texture model. This experiment has been carried out in three different environments and they are "Original Textures", "Histogram-equalized Textures" and "Histogram-equalized and Randomly-rotated Textures". For this experiment, 20 different texture images are selected from the Brodatz album and they are shown in Fig. 7. All the selected texture images have the size of $640 \times 640$ pixels and represent a texture class. Each texture image is partitioned into fifty sub-images with the size of $64 \times 64$ pixels. So there were 1000 test samples considered for this experiment in total. In this experiment, for the test of "Original Textures", no histogram equalization and no random rotation were applied to both model and test samples. For the test of "Histogram-equalized Textures", histogram equalization was performed on both model samples as well as test samples. Every histogram equalized image in the model samples and test samples are rotated by a random rotation angle between $0^{\circ}$ and $360^{\circ}$ in the case of "Histogram-equalized and Randomly-rotated Textures" test. The classification process was repeated 5 times independently and the average classification accuracies of texture models, Local Binary Patterns (LBP), Local Ternary Patterns (LTP) and Optimized Local Ternary Patterns (OLTP) are calculated and tabled in Table 6. According to the experimental results, the highest classification accuracy in all the environments is achieved by the proposed texture model only.

\subsection{Experiment \#4}

In this experiment, the classification performance of the proposed texture method OLTP is evaluated under the noisy environment. Here also, the sample images that are shown in Fig. 7 are used and each texture sample images were corrupted by the zero mean additive Gaussian noise with four different Signal-to-Noise Ratios (SNR). All the selected sample texture images have the size of $640 \times 640$ pixels and represent a texture class. Each texture image was partitioned into fifty sub-images with the size of $64 \times 64$ pixels. So in total, 1000 test samples were considered for this experiment.

In this experiment also, in addition to the proposed texture model OLTP, two more texture models namely, Local Binary Patterns (LBP) and Local Ternary Patterns (LTP) are considered for the comparative analysis. The classification process was repeated 5 times independently and the average classification accuracies are calculated and tabled in Table 7. From Table 7, it can be understood that, until the SNR value is maintained as 35 , all the texture models provide good average classification accuracies because at this level of SNR value, noise effect is too small to distort the image. However, when the SNR value drops below 35, it can be noticed that due to the increase in noise power, all methods have significant drop in the classification accuracies. But even when the value of SNR is maintained as 5, the proposed method effectively performed to achieve sensible classification accuracy ( $83.45 \%)$.

For further clarification, again the same sample texture images of Fig. 7 were partitioned into $25 \mathrm{sub}$ images with the size of $128 \times 128$ pixels. In total, 500 test samples were considered for this sub experiment. Again all the sub images were corrupted by the zero mean additive Gaussian noise with 3 different signal-to-noise ratios $(\mathrm{SNR}=5, \mathrm{SNR}=15$ and $\mathrm{SNR}=30)$. This classification process was also repeated 5 times to consider the classification performance of various texture models (LBP, LTP and OLTP) and the average classification accuracies are displayed in a graphical format in Fig. 8. It is proved that the proposed texture model OLTP is robust in the noisy environment since it maintains its classification performance superior to all other texture models even under heavy noisy conditions. From Fig. 8, it is also observed that, the classification accuracy becomes higher when larger size test samples were used and when the value of SNR becomes low or when the noise becomes severe, the classification performance degrades accordingly. 


\section{DISCUSSION}

\subsection{Robust to Gray-Scale Variation}

In a digital image, when the illumination varies, it causes sudden changes in the grey-scale of the pixel values. The grey-scale invariant property in texture analysis is very much important in the sense that illuminations variations either in local level or global level cannot be avoided in digital images due to various reasons. It is known that the proposed texture model OLTP uses a scaling factor $(\sigma)$ and the centre pixel of the local neighborhood region for which the pattern string is calculated, for encoding the patterns. The availability of scaling factor $(\sigma)$ helps OLTP to use a more tolerable and dynamic threshold range for encoding the patterns. So, when there is a sudden change in the surrounding pixel values of the image due to the illumination variation, correspondingly there is a change in the threshold range to accommodate these changes. This is the reason for the grey-scale invariant property of the proposed texture model OLTP. But this is not the case in LTP, as it uses a fixed threshold to do the encoding. This is clearly explained in the Fig. 9.

To test the grey-scale invariant property of the proposed texture model, grey-scale transform is applied to the image pixel values. Figure 9a shows the original image pixel values before the grey-scale transform is applied and Fig. 9a1 shows the image pixel values after the grey-scale transform (multiplied by constant ' 3 ') is applied. Figure 9b-d are the LBP, LTP and OLTP encodings of the original image pixel values respectively, whereas Fig. 9 b1, c1 and d1 are the LBP,

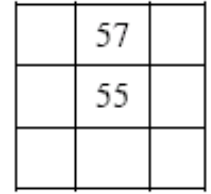

(a)

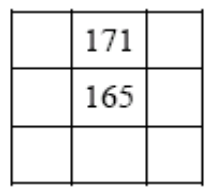

(a1)

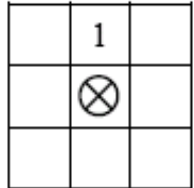

(b)

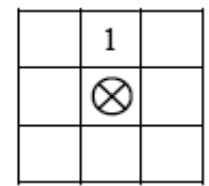

(b1)

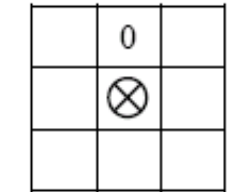

(c)

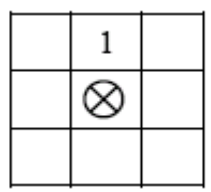

(c1)
LTP and OLTP encodings of the grey-scale transformed version of the original image pixel values respectively. Since both OLTP encodings for the original image pixel values and the grey-scale transformed one are same (Fig. $9 \mathbf{d}$ and d1), it is proved that the proposed texture model, OLTP is grey-scale invariant method. Another texture model LBP is also invariant to gray-scale transforms which can be easily observed from the same LBP encodings of original image pixel values and grey-scale transformed pixel values (Fig. 9b and b1). When LTP is considered, Fig. 9c and c1 are different that leads to a conclusion that LTP is not robust to grey-scale variance.

\subsection{Robust to Rotation Variation}

This proposed model OLTP is robust in terms of rotation variation also. As, OLTP texture model uses the optimal patterns which are nothing but the subset of rotation invariant uniform patterns, it is understood that the proposed texture model should be rotation invariant method.

The results of Experiment \#2, which was experimented in the toughest condition of unrotated model samples and rotated test samples, substantiate this interpretation. From the observations, it is noted that number of patterns play an important role in the texture models. It is also observed that when larger number of patterns is used for texture description, more likely the misclassification of patterns in the encoding occurs, especially when the rotation is applied to the digital images. As expected, upon rotation, the proposed texture method OLTP yielded better classification accuracy than other compared texture methods LBP and LTP, because it uses only less number of patterns (optimal patterns).

Fig. 9. Achieving Grey-scale Invariance: Comparision of LBP, LTP and OLTP texture methods (a) original image pixel values in a $3 \times 3$ neighborhood, (b), (c), (d) LBP, LTP, OLTP encodings of (a) respectively, (a1) grey-scale transformed pixels (multiplied by constant ' 3 '), (b1) LBP encoding after grey-scale transform, (c1) LTP encoding after grey-scale transform, (d1) OLTP encoding after grey-scale transform 


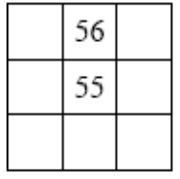

(a)

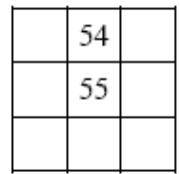

(a1)

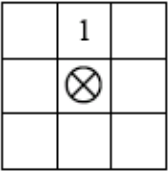

(b)

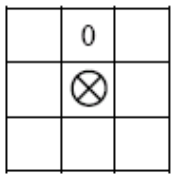

(b1)

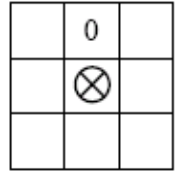

(c)

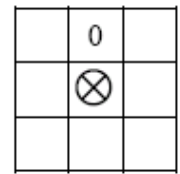

(c1)

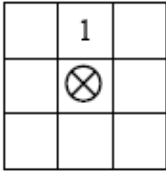

(d)

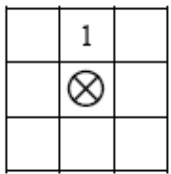

(d1)

Fig. 10. Achieving noise resistance: Comparision of LBP, LTP and OLTP texture methods (a) original image pixel values in a $3 \times 3$ neighborhood, (b) LBP encoding of (a), (c) LTP encoding of (a), (d) OLTP encoding of (a), (a1) image pixel values after noise nclusion, (b1) LBP encoding after noise inclusion, (c1) LTP encoding after noise inclusion, (d1) OLTP encoding after noise inclusion

\subsection{Less Sensitive to Histogram Equalization}

By considering Experiment \#3, it can be noted that unlike other compared texture models, there was no considerable drop in the classification accuracy in the proposed OLTP approach, when the image rotation with histogram equalization was applied. So, in this attribute also, proposed OLTP method outperforms other two compared methods (LBP and LTP) and it has been proved that proposed OLTP method is robust to histogram equalization too.

\subsection{Robustness to Noise}

Performing texture classification under a noisy environment can examine the robustness of any texture model against noise in real world applications. Since the proposed texture model OLTP is using a more flexible and dynamic threshold range which is based on the value of the centre pixel with the help of a scaling factor, it is also more robust to noise. In the case of LBP, though it is grey-scale invariant, it is sensitive to noise especially in the more uniform regions because it uses a fixed threshold that is based on exactly at the value of center pixel. As LTP is using a small fixed range as the threshold, it is not sensitive against the noise. This concept is clearly shown in the Fig. 10. Figure 10a shows the original pixel values prior to the application of noise and Fig. 10a1 shows the pixel values after the noise is applied. Figure 10b-d are the LBP, LTP and OLTP encodings of the original image pixel values respectively, whereas Fig. 10b1, c1 and d1 are the LBP, LTP and OLTP encodings of the noise included version of the original image pixel values respectively. Since both OLTP encodings for the original pixel values and the noise included one are same (Fig. 10d and d1), it is understood that the proposed texture model OLTP is a noise resistant approach. Another texture model LTP is also resistant to noise which can be easily observed from the same LTP encodings of original values and noise included pixel values (Fig. 10c and c1). When LBP is considered, Fig. 10b and b1 are different that leads to a conclusion that LBP is not robust to noise. Experimental results (Experiment \#4) also validate this point that the proposed texture model OLTP is robust to noise.

The following sentences summarize and discuss the implications of the experimental results with the focus on the significance of the salient features of proposed texture model OLTP. Three important and already established texture models have been involved in the comparative analysis with the proposed texture model OLTP and they are Texture Spectrum (TS), Local Binary Patterns (LBP) and Local Ternary Patterns (LTP). Like the proposed model OLTP, TS model also uses a ternary level of comparison in a $3 \times 3$ local neighborhood and it gives good classification results but at the cost of more memory space since it is using more number of patterns (6561) than the proposed method OLTP. Since TS method uses very large number of patterns to describe the texture in an image, the computational time is very high and it cannot be a useful tool for the applications, where time is a critical factor. Further, basically Texture Spectrum (TS) model is not a rotation and grey-scale invariant model. But at the same time, another texture model LBP, though it uses binary level of comparison to encode the patterns, it is more robust against gray-scale variations and rotation variations. Since LBP method uses only 10 numbers of patterns for texture representation, it needs less memory requirement and less execution time, 
but it is inefficient to characterize the local regions. Though LBP texture model is grey-scale invariant, it is sensitive to noise and on the other hand, another recently introduced texture model LTP, is resistant to noise but it is not a grey-scale invariant method.

Proposed texture method OLTP is performing better because, it uses 24 numbers of optimal patterns which is sufficiently large enough to characterize the local spatial relationship of a texture. Number of patterns, used in the proposed texture model OLTP, is more than insufficient LBP model and less than expensive TS model. Regarding LTP, though it gave nearly equal performance in some experiments like OLTP, it uses almost double the number of patterns compared to OLTP. In some times, using more number of patterns to describe the texture information may lead to poor performance and this is true in the case of LTP. This is the reason for LTP texture model for giving lower classification accuracy in some experiments in this study, compared to proposed texture model. Moreover, it is also experimentally proved that the optimal patterns of the proposed texture model OLTP are the real discriminating and active patterns among the uniform patterns of LTP model. So, it is concluded that, optimal patterns of the proposed texture model OLTP are the fundamental properties of a texture in a $3 \times 3$ local neighborhood of a digital texture image.

\section{CONCLUSION}

This study proposed a new spatial method of texture modeling approach called Optimized Local Ternary Patterns (OLTP). This study also introduced a new concept called, "Level of Optimality", which is very simple and computationally efficient, to select the optimal patterns among the uniform patterns. On one hand, like conventional LBP approach, the proposed method OLTP has the properties of rotation invariant and gray-scale invariant. On the other hand, like LTP, it has the ability to withstand against the noise also. Further, it was also experimentally proved that this newly proposed texture model is histogram equalization invariant. The quality of the proposed approach was validated with many numbers of experiments to prove that this OLTP is robust to grey-scale variation, rotation variation, histogram equalization and noise. This proposed OLTP texture method on one side gives better classification accuracy than recently introduced LTP texture approach. On the other side, it uses only half the number of uniform patterns of LTP method. It was experimentally proved that the optimal patterns of the proposed texture model OLTP are the fundamental properties of textures and they are the dominant patterns in the uniform patterns of the LTP model. The experimental results show that the proposed method outperforms the other widely used texture models in terms of the classification accuracy under different experimental conditions. Since the proposed OLTP is robust in every aspect it can be a good replacement for both LBP and LTP.

Regarding the future work, the proposed texture model OLTP can be tested for image texture segmentation problems. The proposed approach can also be checked for color texture images. This study uses only G-Statistics similarity measure in the classification algorithm and other similarity measures like Euclidean distance, Manhattan distance can also be used for further study.

\section{REFERENCES}

Ahmed, F., E. Hossain, A.S.M.H. Bari and A.S.M. Shihavuddin, 2011. Compound local binary pattern (CLBP) for robust facial expression recognition. Proceedings of the IEEE 12th International Symposium on Computational Intelligence and Informatics, Nov. 21-22, IEEE Xplore Press, Budapest, pp: 391-395. DOI: 10.1109/CINTI.2011.6108536

Ahonen, T. and M. Pietikainen, 2007. Soft histograms for local binary patterns. Proceedings of the Finnish Signal Processing Symposiumm, (FSPS' 07), Finland, Oulu, pp: 1-4.

Brodatz, P., 1966. Textures: A Photographic Album for Artists and Designers. 1st Edn., Dover Publications, New York, ISBN-10: 0486216691, pp: 112.

Coggins, J.M. and A.K. Jain, 1985. A spatial filtering approach to texture analysis. Patt. Recog. Lett., 3: 195-203. DOI: 10.1016/0167-8655(85)90053-4

Daugman, J.G., 1980. Two-dimensional spectral analysis of cortical receptive field profiles. Vis. Res., 20: 847-856. DOI: 10.1016/0042-6989(80)90065-6

Fu, K.S., 1982. Syntactic Pattern Recognition and Applications. 1st Edn., Prentice Hall, Englewood Cliffs, N.J., ISBN-10: 0138801207, pp: 596.

Galloway, M.M., 1975. Texture analysis using gray level run lengths. Comput. Graphics Image Proces., 4: 172-179. DOI: 10.1016/S0146-664X(75)80008-6

Haralick, R.M., K. Shanmugam and I. Dinstein, 1973. Textural features for image classification. IEEE Trans. Syst. Man Cybernetics, 3: 610-621. DOI: 10.1109/TSMC.1973.4309314

Heeger, D.J. and J.R. Bergen, 1995. Pyramid-based texture analysis/synthesis. Proceedings of the 22nd Annual Conference on Computer Graphics and Interactive Techniques, Aug. 06-11, ACM Press, USA., pp: 229-238. DOI: 10.1145/218380.218446 
Hussain, S. and B. Triggs, 2010. Feature sets and dimensionality reduction for visual object detection. Proceedings of the British Machine Vision Conference, Aug. 31-Sep. 3, BMVA Press, UK., pp: 112.1-112.10. DOI: $10.5244 /$ C.24.112

Liao, W.H., 2010. Region description using extended local ternary patterns. Proceedings of the 20th International Conference on Pattern Recognition, Aug. 23-26, Istanbul, Turkey, pp: 1003-2006. DOI: 10.1109/ICPR2010.251

Liu, Y., Y. Tsin and W.C. Lin, 2005. The promise and perils of near-regular texture. Int. J. Comput. Vis., 62: 145-159. DOI: 10.1007/s11263-005-4639-0

Liu, Y.Y., M. Chen, H. Ishikawa, G. Wollstein and J.M. Rehg, 2011. Automated macular pathology diagnosis in retinal OCT images using multi-scale spatial pyramid and local binary patterns in texture and shape encoding. Med. Image Anal., 15: 748759. DOI: 10.1016/j.media.2011.06.005

Matsuyama, T., S. Miura and M. Nagao, 1983. Structural analysis of natural textures by Fourier transformation. Comput. Vis. Graphics Image Process., 24: 347-362. DOI: 10.1016/0734-189X(83)90060-9

Moasheri, B.B.M. and S. Azadinia, 2011. A new voting approach to texture defect detection based on multiresolutional decomposition. World Acad. Sci., Eng. Technol., 73: 657-661.

Nanni, L., S. Brahnam and A. Lumini, 2010. A local approach based on a local binary patterns variant texture descriptor for classifying pain states. Exp. Syst. Appl., 37: 7888-7894. DOI: 10.1016/j.eswa.2010.04.048

Ojala, T. and M. Pietikainen, 2004. Texture Classification. Machine Vision and Media Processing Unit University of Oulu, Finland.

Ojala, T., M. Pietikainen and D. Harwood, 1996. A comparative study of texture measures with classification based on featured distributions. Patt. Recog., 29: 51-59. DOI: 10.1016/00313203(95)00067-4

Ojala, T., M. Pietikainen and T. Maenpaa, 2002. Multiresolution gray-scale and rotation invariant texture classification with local binary patterns. IEEE Trans. Patt. Anal. Mach. Intell., 24: 971-987. DOI: 10.1109/TPAMI.2002.1017623

Rodriguez, Y. and S. Marcel, 2006. Face authentication using adapted local binary pattern histograms. Proceedings of the 9th European Conference on Computer Vision (ECCV' 06), LNCS, Springer, Heidelberg, pp: 321-332. DOI: 10.1007/11744085_25
Sokal, R.R. and F.J. Rohlf, 2009. Introduction to Biostatistics. 2nd Edn., Dover Publications, New York, ISBN-10: 9780486469614, pp: 363.

Suguna, R. and P. Anandhakumar, 2010. A rotation invariant pattern operator for texture characterization. Int. J. Comput. Sci. Netw. Security, 10: $120-129$.

Suruliandi, A. and K. Ramar, 2008. local texture patterns-a univariate texture model for classification of images. Proceedings of the 16th International Conference on Advanced Computing and Communications, Dec. 1417, IEEE Xplore Press, Chennai, pp: 32-39. DOI: 10.1109/ADCOM.2008.4760424

Tan, X. and B. Triggs, 2010. Enhanced local texture feature sets for face recognition under difficult lighting conditions. IEEE Trans. Image Proces., 19: 1635-1650. DOI: 10.1109/TIP.2010.2042645

Trefny, J. and J. Matas, 2010. Extended set of local binary patterns for rapid object detection. Proceedings of the Computer Vision Winter Workshop, Feb. 3-5, Czech Pattern Recognition Society, pp: 1-7.

Tsatsanis, M.K. and G. B. Giannakis, 1992. Object and texture classification using higher order statistics. IEEE Trans. Patt. Anal. Machine Intell., 14: 733750. DOI: $10.1109 / 34.142910$

Tuceryan, M. and A.K. Jain, 1999. Texture Analysis. In: Handbook of Pattern Recognition and Computer Vision, Chen, C.H., L.F. Pau, P.S.P. Wang and S.P. Wang (Eds.), World Scientific, Singapore, ISBN-10: 9810230710, pp: 207-248.

Weszka, J.S., C.R. Dyer and A. Rosenfeld, 1976. A comparative study of texture measures for terrain classification. IEEE Trans. Syst. Man Cybernetics, 6: 269-285. DOI:10.1109/TSMC.1976.5408 777

Xia, Y., D. Feng and R. Zhao, 2006. Morphology-Based multifractal estimation for texture segmentation. IEEE Trans. Image Process., 15: 614-623. DOI: 10.1109/TIP.2005.863029

Zheng, Y., C. Shen, R. Hartley and X. Huang, 2011. Pyramid center-symmetric local binary/trinary patterns for effective pedestrian detection. Proceedings of the 10th Asian Conference on Computer Vision Volume Part IV, Nov. 8-12, Queenstown, New Zealand, pp: 281-292. DOI: 10.1007/978-3-642-19282-1_23

Zhu, S.C., Y.N. Wu and D. Mumford, 1998. Filters, random fields and maximum entropy (frame): Towards a unified theory for texture modeling. Int. J. Comput. Vis., 27: 107-126. DOI: 10.1023/A:1007925832420 